\title{
Recent Results of Source Function Imaging from AGS through CERN SPS to RHIC
}

\author{
Roy A. Lacey \\ Dept of Chemistry, SUNY Stony Brook, Stony Brook, NY 11794, USA
}

Received on 19 January, 2007

\begin{abstract}
Recent femtoscopic measurements involving the use of an imaging technique and a newly developed moment analysis are presented and discussed. We show that this new paradigm allows robust investigation of reaction dynamics for which the sound speed $c_{s} \neq 0$ during an extended hadronization period. Source functions extracted for charged pions produced in $\mathrm{Au}+\mathrm{Au}$ and $\mathrm{Pb}+\mathrm{Pb}$ collisions show non-Gaussian tails for a broad selection of collision energies. The ratio of the RMS radii of these source functions in the out and side directions are found to be greater than 1 , suggesting a finite emission time for pions.
\end{abstract}

Keywords: Correlation; Interferometry; Intensity interferometry; Cartesian harmonics

\section{INTRODUCTION}

Lattice calculations of the thermodynamical functions of quantum chromodynamics (QCD) indicate a rapid transition from a confined hadronic phase to a chirally symmetric deconfined quark gluon plasma (QGP), for a critical temperature $T_{c} \sim 170 \mathrm{MeV}$ [1]. The creation and study of this QGP is one of the most important goals of current ultra-relativistic heavy ion research [2]. Central to this endeavor, is the question of whether or not the QGP phase transition is first or$\operatorname{der}(\Delta T=0), 2^{\text {nd }}$ order, or a rapid crossover reflecting an increase in the entropy density associated with the change from hadronic $\left(d_{H}\right)$ to quark and gluon $\left(d_{Q}\right)$ degrees of freedom. Currently, lattice calculations seem to favor a rapid crossover $[3,4]$ i.e. $\Delta T \neq 0$ for the transition.

An emitting system which undergoes a sharp first order QGP phase transition is predicted to show a larger space-time extent, than that for a purely hadronic system $[5,6]$. Here, the rationale is that the transition to the QGP "softens" the equation of state (ie. the sound speed $c_{S} \sim 0$ ) in the transition region, and this delays the expansion and considerably prolongs the lifetime of the system. That is, matter-flow through this soft region of energy densities during the expansion phase, will temporarily slow down and could even stall under suitable conditions.

To explore this prolonged lifetime, it has been common practice to measure the widths of emission source functions (assumed to be Gaussian) in the out- side- and long-direction $\left(R_{\text {out }}, R_{\text {side }}\right.$ and $R_{\text {long }}$ ) of the Bertsch-Pratt coordinate system [7-10]. For such extractions, the Coulomb effects on the correlation function are usually assumed to be separable [11] as well. The ratio $R_{\text {out }} / R_{\text {side }}>1$, is expected for systems which undergo a strong first order QGP phase transition $[5,6]$. This is clearly illustrated in Fig. 1, where the values obtained from a hydrodynamical model calculation [6] are plotted as a function of energy density (in units of $T_{c} s_{c} ; s$ is the entropy density). These rather large ratios (especially those in Fig. 1a and c) have served as a major motivating factor for experimental searches of a prolonged lifetime at several accelerator facilities [7-10, 12-14].

An interesting aspect of Fig. 1 is the strong dependence of $R_{\text {out }} / R_{\text {side }}$ on the width of the transition region (i.e. $\Delta T \neq$ $0)$. A comparison of Figs. 1a and b shows that this ratio is

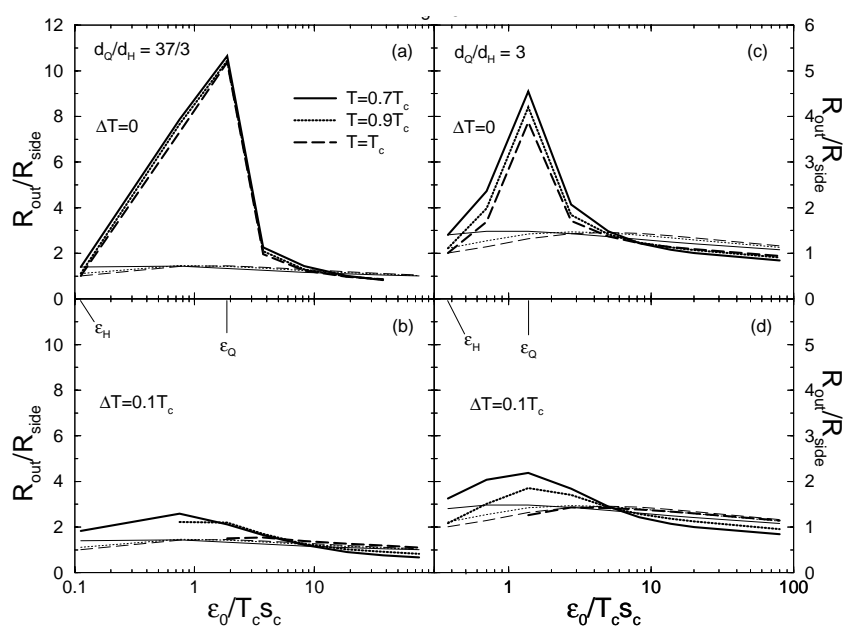

FIG. 1: $R_{\text {out }} / R_{\text {side }}$ as a function of the initial energy density (in units of $T_{C} s_{C}$ ) for an expanding fireball. (a,b) are for $d_{Q} / d_{H}=37 / 3,(\mathrm{c}, \mathrm{d})$ for $d_{Q} / d_{H}=3$. The thick lines in $(\mathrm{a}, \mathrm{c})$ are for $\Delta T=0$, in $(\mathrm{b}, \mathrm{d})$ for $\Delta T=0.1 T_{c}$. Thin lines show results for an ideal gas case. Solid lines show results for $T=0.7 T_{c}$, dotted for $T=0.9 T_{c}$, dashed for $T=T_{c}$. The figure is taken from Ref. [6].

considerably reduced (by as much as a factor of four) when the calculations are performed for $\Delta T=0.1 T_{c}$. Thus, the spacetime extent of the emitting system is rather sensitive to the reaction dynamics and whether or not the transition is a cross over. It has also been suggested that the shape of the emission source function can provide signals for a second order phase transition and whether or not particle emission occurs near to the critical end point in the QCD phase diagram [15].

\section{REACTION DYNAMICS AT RHIC}

Experimental evidence for the creation of locally equilibrated nuclear matter at unprecedented energy densities at RHIC, is strong [16-23]. Jet suppression studies indicate that the constituents of this matter interact with unexpected strength and this matter is almost opaque to high energy partons [24, 25].

Elliptic flow measurements [26, 27] validate the predictions 


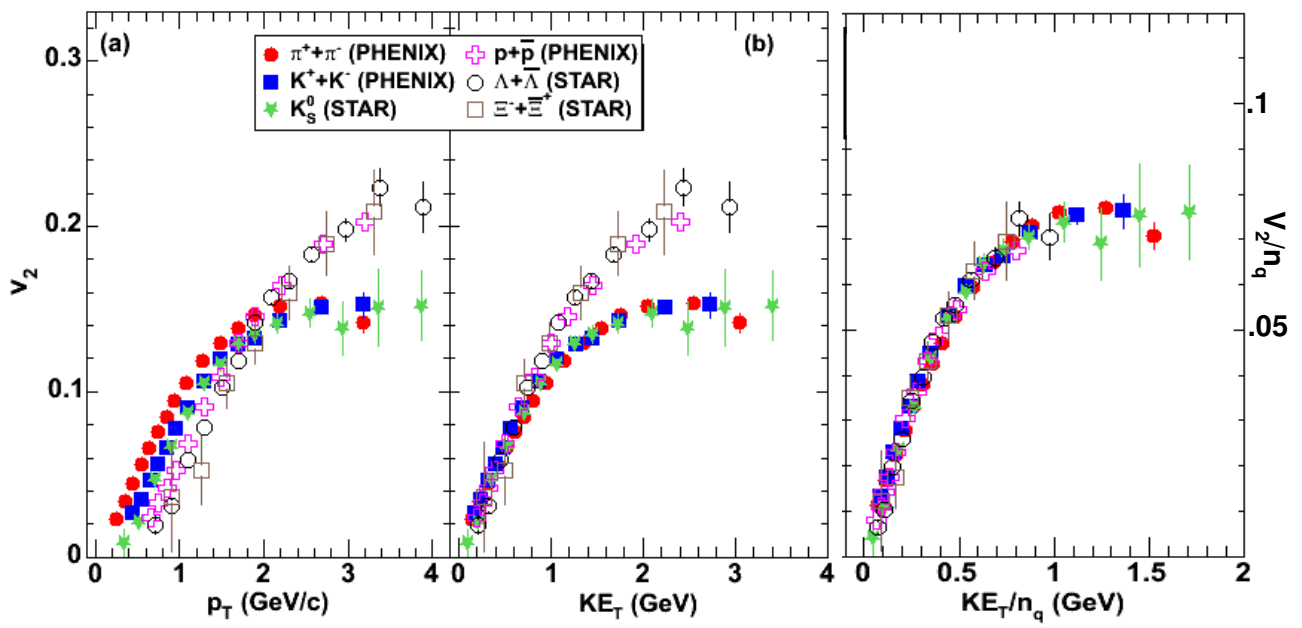

FIG. 2: (Color online) $v_{2}$ vs. $p_{T}$ (left panel) and $K E_{T}$ (middle panel). The scaled results in the right panel is obtained via $n_{q}$ scaling of the data shown in the middle panel. Results are shown for several particle species produced in minimum bias Au+Au collisions at $\sqrt{s_{N N}}=200 \mathrm{GeV}$ [26].

of perfect fluid hydrodynamics for the scaling of the elliptic flow coefficient $v_{2}$ with eccentricity $\varepsilon$, system size and transverse kinetic energy $\mathrm{KE}_{T}$ [28-30]; they also indicate the predictions of valence quark number $\left(n_{q}\right)$ scaling [31-33]. The result of such scaling is illustrated in Fig. 2c; it shows that, when plotted as a function of the transverse kinetic energy $\mathrm{KE}_{T}$ and scaled by the number of valence quarks $n_{q}$ of a hadron ( $n_{q}=2$ for mesons and $n_{q}=3$ for baryons), $v_{2}$ shows a universal dependence for a broad range of particle species $[26,27,34]$. This has been interpreted as evidence that hydrodynamic expansion of the QGP occurs during a phase characterized by (i) a rather low viscosity to entropy ratio $\eta / s$ $[20,22,23,34]$ and (ii) independent quasi-particles which exhibit the quantum numbers of quarks [31-36].

The scaled $v_{2}$ values shown in Fig. 2c allow an estimate of $c_{s}$ because the magnitude of $v_{2} / \varepsilon$ depends on the sound speed [30]. One such estimate [26,27] gives $c_{s} \sim 0.35 \pm 0.05$; a value which suggests an effective equation of state (EOS) which is softer than that for the high temperature QGP [3]. It however, does not reflect a strong first order phase transition in which $c_{s} \sim 0$ during an extended hadronization period. This sound speed is also compatible with the fact that $v_{2}\left(p_{T}\right)$ is observed to saturate in $\mathrm{Au}+\mathrm{Au}$ collisions for the collision energy range $\sqrt{s_{N N}}=60-200 \mathrm{GeV}$ [37].

Femtoscopic measurements involving the use of the Bowler-Sinyukov 3D HBT analysis method [in Bertsch-Pratt coordinates], have been used to probe for long-range emissions from a possible long-lived source [7-10, 12-14]. The observed RMS-widths for each dimension of the emission source $R_{\text {long }}, R_{\text {side }}$ and $R_{\text {out }}$, show no evidence for such emissions. That is, $R_{\text {out }} / R_{\text {side }} \sim 1.0$. It is somewhat paradoxical that these observations have been interpreted as a femtoscopic puzzle [7] - "the HBT puzzle" - despite the fact that they clearly reflect a rich and complex set of thermodynamic trajectories for which $c_{s} \neq 0$ (or $\Delta T \neq 0$ ) during an extended hadronization period.

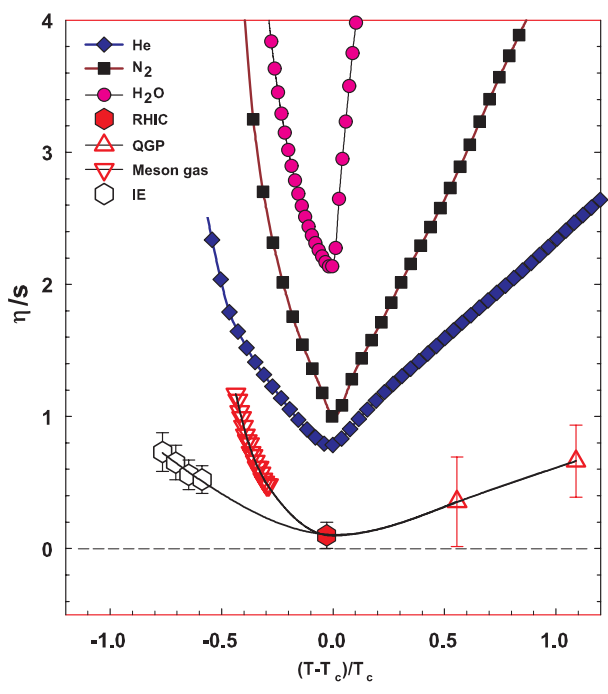

FIG. 3: (Color online) $\eta / s$ vs $\left(T-T_{c}\right) / T_{C}$ for several substances as indicated. The calculated values for the meson-gas have an associated error of $\sim 50 \%$ [38]. The lattice $\mathrm{QCD}$ value $T_{c}=170 \mathrm{MeV}$ [4] is assumed for nuclear matter. The lines are drawn to guide the eye.

Hints for even more complicated reaction dynamics is further illustrated via a comparison of $\eta / s$ values for nuclear, atomic and molecular substances in Fig. 3. It shows the observation that for atomic and molecular substances, the ratio $\eta / s$ exhibits a minimum of comparable depth for isobars passing in the vicinity of the liquid-gas critical point $[39,40]$. When an isobar passes through the critical point (as in Fig. 3), the minimum forms a cusp at $T_{c}$; when it passes below the critical point, the minimum is found at a temperature below $T_{c}$ (liquid side) but is accompanied by a discontinuous change across the phase transition. For an isobar passing above the critical point, 
a less pronounced minimum is found at a value slightly above $T_{c}$. The value $\eta / s$ is smallest in the vicinity of $T_{c}$ because this corresponds to the most difficult condition for the transport of momentum [40].

Given these observations, one expects a broad range of trajectories in the $\left(T, \mu_{B}\right)$ plane for nuclear matter, to show $\eta / s$ minima with a possible cusp at the critical point $\left(\mu_{B}\right.$ is the baryon chemical potential). The exact location of this point is of course not known, and only coarse estimates of where it might lie are available. The open triangles in the figure show calculated values for $\eta / s$ along the $\mu_{B}=0, n_{B}=0$ trajectory. For $T<T_{C}$ the $\eta / s$ values for the meson-gas show an increase for decreasing values of $T$. For $T$ greater than $T_{c}$, the lattice results [41] indicate an increase of $\eta / s$ with $T$, albeit with large error bars.

These trends suggest a minimum for $\eta / s$, in the vicinity of $T_{c}$, whose value is close to the conjectured absolute lower bound $\eta / s=1 / 4 \pi$. Consequently, it is tempting to speculate that it is the minimum expected when the hot and dense QCD matter produced in RHIC collisions follow decay trajectories which are close to the QCD critical end point (CEP). Such trajectories could be readily followed if the CEP acts as an attractor for thermodynamic trajectories of the decaying matter $[42,43]$.

The above insights on the reaction dynamics of hot and dense QCD matter, strongly suggest that full utility of the femtoscopic probe requires detailed measurements and theoretical investigations of both the shape and the space-time character of emission source functions. Here, it should be stressed that a good understanding of the space-time evolution of the QGP is crucial. This is because, in contrast to other signals, it is derived from the influence of the equation of state on the collective dynamical evolution of the system. This is less clear-cut for many other proposed signals [44, 45].

\section{SOURCE IMAGING: A FEMTOSCOPIC PARADIGM SHIFT}

\section{A. 1D source functions via imaging}

An initial strategy has been to extract the "full" distribution of the 1D emission source function [46]. The 1D Koonin-Pratt equation [47]:

$$
C(q)-1=4 \pi \int d r r^{2} K_{0}(q, r) S(r),
$$

relates this source function $S(r)$ (i.e. the probability of emitting a pair of particles at a separation $r$ in the pair center of mass (PCMS) frame) to the $1 \mathrm{D}$ correlation function $C(q)$. The angle-averaged kernel $K_{0}(q, r)$ encodes the the final state interaction (FSI) which is given in terms of the final state wave function $\Phi_{\mathbf{q}}(\mathbf{r})$, as $K_{0}(q, r)=\frac{1}{2} \int d\left(\cos \left(\theta_{\mathbf{q}, \mathbf{r}}\right)\right)\left(\left|\Phi_{\mathbf{q}}(\mathbf{r})\right|^{2}-1\right)$, where $\theta_{\mathbf{q}, \mathbf{r}}$ is the angle between $\mathbf{q}$ and $\mathbf{r}$ [48]

The model-independent imaging technique of Brown and Danielewicz $[48,49]$ is used to invert Eq. 1 to obtain $S(r)$. In brief, it utilizes a numerical calculation of the two particle wave function (including FSI) to produce an inversion matrix
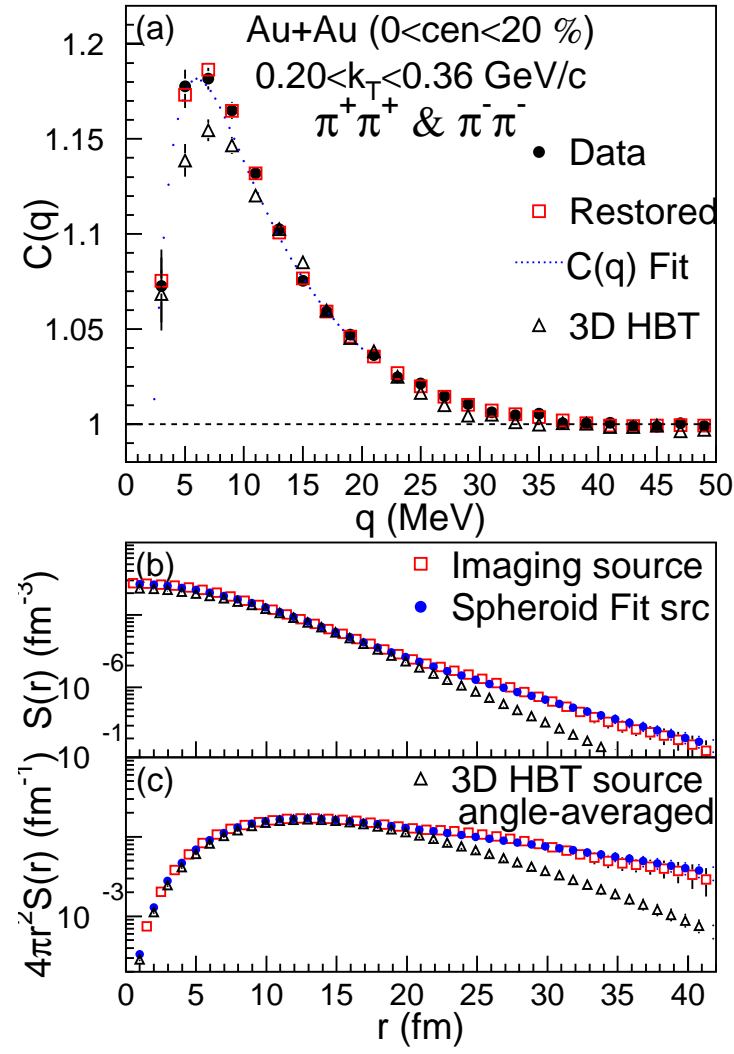

FIG. 4: (Color online) (upper, a) (filled circles) Correlation function, $\mathrm{C}(\mathrm{q})$ for $\pi^{+} \pi^{+}$and $\pi^{-} \pi^{-}$pairs; (open squares) restored correlation from imaging technique; (dotted line) direct correlation fitting; (open triangles) 1D angle-averaged correlation of 3D correlation function. (lower) 1D source function (b) $\mathrm{S}(\mathrm{r})$ and (c) $4 \pi r^{2} \mathrm{~S}(\mathrm{r})$ : (open squares) imaging; (filled circles) spheroid fit to correlation function; (open triangles) angle-averaging of 3D-Gaussian source function. The figure is reproduced from Ref. [46].

that operates on the measured correlation function to give the associated source function. A detailed description of the procedure for robust measurement of the correlation functions is given in these proceedings [50].

The open squares in Fig. 4(b) show the 1D source image obtained from the correlation function (filled circles) presented in Fig. 4(a). The open squares in Fig. 4(a) show the corresponding restored correlation function obtained via Eq. 1 with the extracted source function as input. Their consistency serves as a good cross check of the imaging procedure.

Figure 4(b)) indicates a Gaussian-like pattern at small $r$ and an unresolved "tail" at large $r$. For comparison, the source function constructed from the parameters $\left(R_{\text {long }}, R_{\text {side }}, R_{\text {out }}\right.$ and $\lambda$ ), obtained in an earlier 3D HBT analysis [14] is also shown; the associated 3D angle-averaged correlation function is shown in Fig. 4(a). The imaged source function exhibits a more prominent tail than the angle averaged 3D HBT source function. These differences reflect the differences in the associated correlation functions for $q \lesssim 10-15 \mathrm{MeV}$, as shown in Fig. 4(a). This could come from the Gaussian shape assumption employed for $S(r)$ in the 3D HBT analysis. That is, 
the 3D Gaussian fitting procedure by construction is sensitive only to the main component of $S(r)$, and thus would not be capable of resolving fine structure at small-q/large-r.

The radial probabilities $\left(4 \pi r^{2} S(r)\right)$ are also compared in Fig.4(c); they show that the differences are actually quite large. They are clearly related to the long-range contribution to the emission source function.

\section{B. 1D source function via correlation function fitting}

Another approach for 1D source function extraction is to perform direct fits to the correlation function. This involves the determination of a set of values for an assumed source function shape, which reproduce the observed correlation function when the resulting source function is inserted into Eq. 1. The filled circles in Figs. 4(b,c) show the source function obtained from such a fit for a Spheroidal shape [48],

$$
S(r)=\frac{\lambda R_{\mathrm{eff}} \times e^{-\frac{r^{2}}{4 R_{T}^{2}}} \operatorname{erfi}\left(\frac{r}{2 R_{\mathrm{eff}}}\right)}{\left(8 \pi R_{T}^{2} R_{0} r\right)}, \text { for } R_{0}>R_{T}
$$

where $R_{\text {eff }}=1 / \sqrt{\left(1 / R_{T}^{2}-1 / R_{0}^{2}\right)}, R_{T}$ is the radius of the Spheroid in two perpendicular spatial dimensions and $R_{0}=$ $a \times R_{T}$ is the radius in the third spatial dimension; $a$ is a scale factor. The long axis of the Spheroid is assumed to be oriented in the out direction of the Bertsch-Pratt coordinate system. The fraction of pion pairs which contribute to the source $\lambda$, is given by the integral of the normalized source function over the full range of $r$. The source function shown in Figs. 4(b) and 4(c), indicates remarkably good agreement with that obtained via the imaging technique, confirming a long-range contribution to the emission source function.

\section{Origin of the long-range contributions to the 1D source function}

Extraction of the detailed shape of the 1D source function is made ambiguous by the observation that several assumed shapes gave equally good fits which reproduced the observed correlation function. Nonetheless, one can ask whether or not a simple kinematic transformation from the longitudinal comoving system (LCMS) to the PCMS can account for the observed tail? Here, the point is that instantaneous freeze-out of a source with $R_{\text {out }} / R_{\text {side }} \sim 1$ in the LCMS would give a maximum kinematic boost so that $R_{\text {out }} \sim \gamma \times R_{\text {side }}$ in the PCMS ( $\gamma$ is the Lorentz factor). Another possibility could be that the particle emission source is comprised of a central core and a halo of long-lived resonances. For such emissions, the pairing between particles from the core and secondary particles from the halo is expected to dominate the long-range emissions [51] and possibly give rise to a tail in the source function.

Both of these possibilities have been checked [46]; the indications are that the observations can not be explained solely by either one. However, further detailed 3D imaging measurements were deemed necessary to quantitatively pin down the origin of the tail in the source function and to determine its possible relationship to the reaction dynamics.

\section{3D SOURCE IMAGING}

In order to develop a clearer picture of the reaction dynamics across beam energies, an ambitious [ongoing] program was developed to measure 3D source functions in heavy ion collisions from AGS through CERN SPS to RHIC energies. This program exploits the cartesian harmonic decomposition technique of Danielewicz and Pratt $[52,53]$ in conjunction with imaging and fitting, to extract the pair separation distributions in the out-, side- and long-directions. Model comparisons to these distributions are expected to give invaluable insights on the reaction dynamics.

\section{A. Cartesian harmonic decomposition of the 3D correlation function}

Following Danielewicz and Pratt [52], the 3D correlation function can be expressed in terms of correlation moments as:

$$
C(\mathbf{q})-1=R(\mathbf{q})=\sum_{l} \sum_{\alpha_{1} \ldots \alpha_{l}} R_{\alpha_{1} \ldots \alpha_{l}}^{l}(q) A_{\alpha_{1} \ldots \alpha_{l}}^{l}\left(\Omega_{\mathbf{q}}\right),
$$

where $l=0,1,2, \ldots, \alpha_{i}=x, y, z, A_{\alpha_{1} \ldots \alpha_{l}}^{l}\left(\Omega_{\mathbf{q}}\right)$ are cartesian harmonic basis elements $\left(\Omega_{\mathbf{q}}\right.$ is solid angle in $\mathbf{q}$ space) and $R_{\alpha_{1} \ldots \alpha_{l}}^{l}(q)$ are cartesian correlation moments given by

$$
R_{\alpha_{1} \ldots \alpha_{l}}^{l}(q)=\frac{(2 l+1) ! !}{l !} \int \frac{d \Omega_{\mathbf{q}}}{4 \pi} A_{\alpha_{1} \ldots \alpha_{l}}^{l}\left(\Omega_{\mathbf{q}}\right) R(\mathbf{q}) .
$$

In Eqs. 3 and 4, the coordinate axes are oriented so that $\mathrm{z}$ is parallel to the beam (i.e. the long direction), $x$ is orthogonal to $\mathrm{z}$ and points along the total momentum of the pair in the LCMS frame (the out direction), and y points in the side direction orthogonal to both $\mathrm{x}$ and $\mathrm{z}$.

The correlation moments, for each order $l$, can be calculated from the measured 3D correlation function with Eq. 4. Another approach is to truncate Eq. 3 so that it includes only non-vanishing moments and hence, can be expressed in terms of independent moments. For instance, up to order $l=4$, there are 6 independent moments: $R^{0}, R_{x 2}^{2}, R_{y 2}^{2}, R_{x 4}^{4}, R_{y 4}^{4}$ and $R_{x 2 y 2}^{4}$. Here, $R_{x 2}^{2}, R_{y 2}^{2}$, etc is used as a shorthand notation for $R_{x x}^{2}$, $R_{y y}^{2}$, etc. The independent moments can then be extracted as a function of $q$ by fitting the truncated series to the experimental $3 \mathrm{D}$ correlation function with the moments as the parameters of the fit. Moments have been so obtained up to order $l=8$, depending on available statistics. The higher order moments $(l \geq 4)$ are generally found to be small or zero especially for the lower collision beam energies.

Figures 5 - 8 show representative comparisons between the 1D correlation functions, $C(q)$, and the $l=0$ moment $C(q)^{0} \equiv$ $R^{0}(q)+1$ for mid-rapidity particles emitted in $\mathrm{Au}+\mathrm{Au}$ and $\mathrm{Pb}+\mathrm{Pb}$ collisions. For the AGS and SPS measurements, $\left|y_{L}-y_{0}\right|<0.35$, where $y_{L}$ is particle laboratory rapidity and 


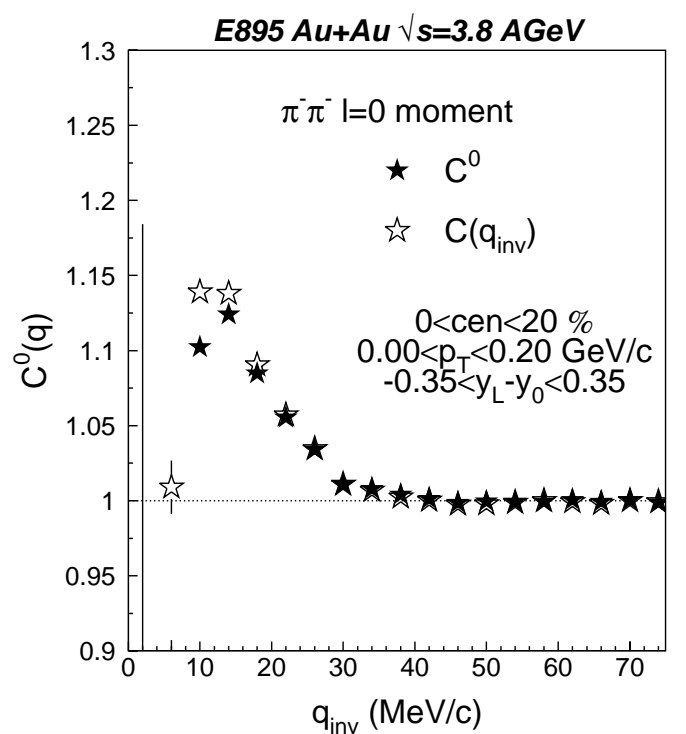

FIG. 5: 1 D correlation function $\mathrm{C}(\mathrm{q})$ (open stars) and $l=0$ moment $C(q)^{0}=R^{0}(q)+1$ (solid stars) for low $p_{T}$ mid-rapidity $\pi^{-} \pi^{-}$pairs from central Au+Au collisions at $\sqrt{s}=3.8 \mathrm{GeV}$.

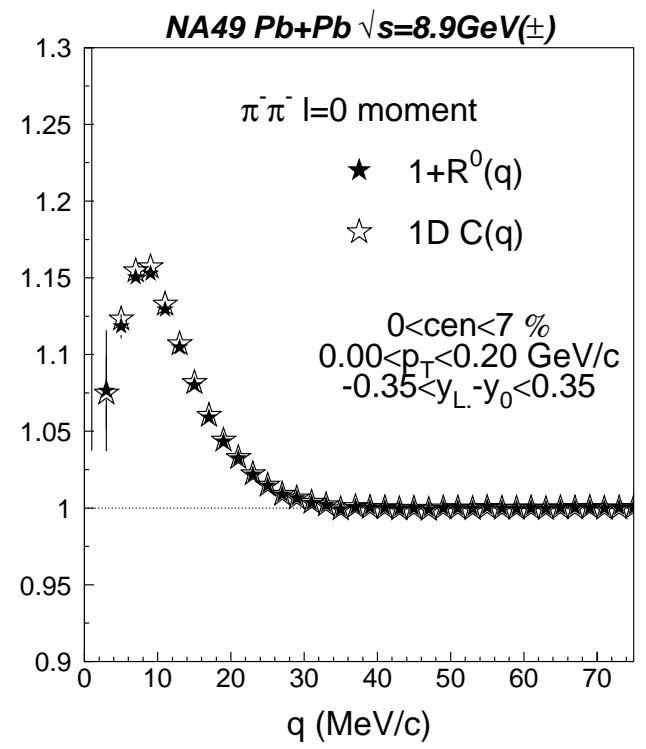

FIG. 6: 1 D correlation function $\mathrm{C}(\mathrm{q})$ (open stars) and $l=0$ moment $C(q)^{0}=R^{0}(q)+1$ (solid stars) for low $p_{T}$ mid-rapidity $\pi^{-} \pi^{-}$pairs from central $\mathrm{Pb}+\mathrm{Pb}$ collisions at $\sqrt{s}=8.9 \mathrm{GeV}$.

$y_{0}$ is CM rapidity. For RHIC measurements $|y|<0.35$. The $p_{T}$ and centrality selections are as indicated in each figure. The $l=0$ moments are in good agreement with the 1D correlation functions; such agreement is expected in the absence of significant pathology, especially those related to the angular acceptance. Therefore, they attest to the reliability of the

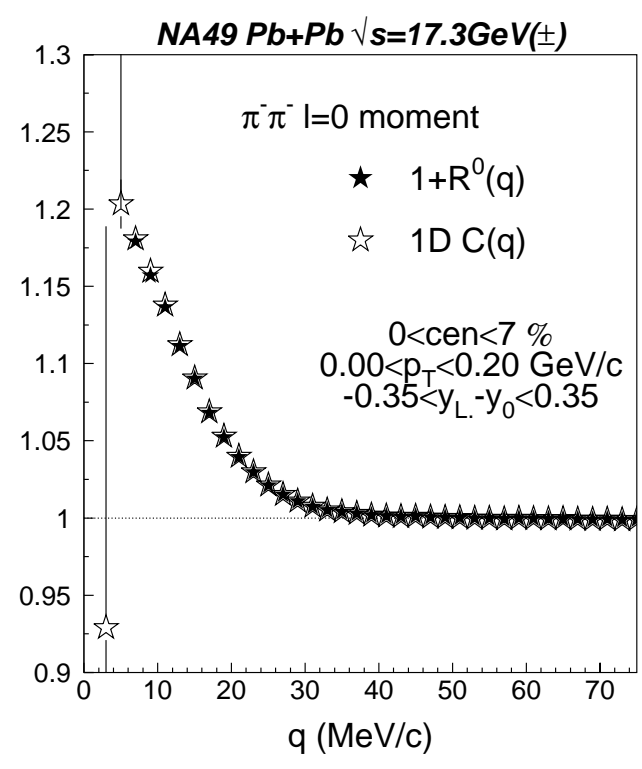

FIG. 7: Same as Fig.6 but for $\sqrt{s}=17.3 \mathrm{GeV}$. Result are averaged over runs with +ve and -ve magnetic fields.

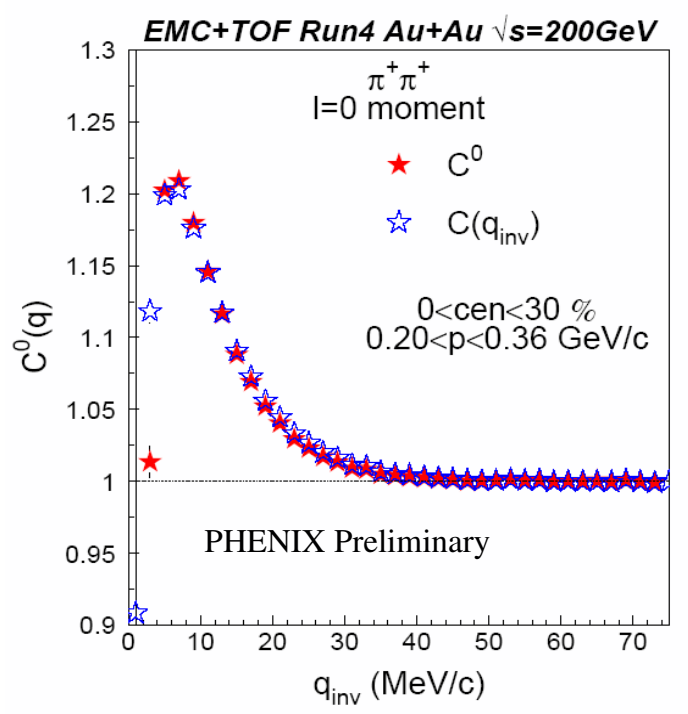

FIG. 8: (Color online) 1D correlation function $\mathrm{C}(\mathrm{q})$ (open stars) and $l=0$ moment $C(q)^{0}=R^{0}(q)+1$ (solid stars) for low $p_{T}$ mid-rapidity $\pi^{-} \pi^{-}$pairs from central Au+Au collisions at $\sqrt{s}=200 \mathrm{GeV}$.

technique used for their extraction. Similarly good agreement was found for all other beam energies and analysis cuts.

Correlation moments for $l=2$ and $l=4$ are shown for $\mathrm{Au}+\mathrm{Au}$ collisions obtained at RHIC, in Fig. 9. The $l=2$ moments show an anti-correlation for $C_{x 2}^{2}(q) \equiv R_{x 2}^{2}(q)$ and positive correlations for $C_{y 2}^{2}(q)$ and $C_{z 2}^{2}(q)$, indicating that 
the emitting source is more extended in the out $(x)$ direction and less so in the side (y) and long ( $\mathrm{z}$ ) directions, when compared to the angle average $C^{0}(q)$ (also see discussion on imaged moments). It is noteworthy that only two of these $l=2$ correlation moments are actually independent, i.e. $C_{x 2}^{2}(q)+C_{y 2}^{2}(q)+C_{z 2}^{2}(q)=0$.

The bottom panel of Fig. 9 shows that rather sizable signals are obtained for $l=4$. Here, it is $C_{x 2 y 2}^{4}$ correlation moment which shows an anti-correlation. Moments were also obtained for $l=6$. Since they were all found to be small, they do not have a significant influence on the shape of the source function.

Figure 10 shows correlation moments for $\mathrm{Pb}+\mathrm{Pb}$ collisions obtained at SPS energies. The top and bottom panels show results for $\sqrt{s}=8.9$ and $17.3 \mathrm{GeV}$ respectively. In each case, the rapidity and centrality selections are the same, but the $p_{T}$ selections are different as indicated. The open squares in the figure represent the result of a simultaneous fit to the independent moments with assumed shapes for the source function as discussed below. Within errors, the magnitudes of the higher order moments were found to be insignificant.

The $R_{x 2}^{2}(q)$ and $R_{y 2}^{2}(q)$ correlation moments in the top panels of Fig. 10 indicate an emission source which is more extended in the out and long directions (compared to $R^{0}(q)$ ). By contrast, the $R_{x 2}^{2}$ and $R_{y 2}^{2}(q)$ correlation moments in the bottom panels indicate positive correlations and hence an emission source with big extension (compared to $R^{0}(q)$ ) in the long direction. In part, this difference is related to a different kinematic factor for the two $p_{T}$ selections.

The correlation moments shown in Figs. 9 and 10 provide the basis for quantitative extraction of the pair separation distributions in the out-, side- and long-directions. This is achieved either by fitting the moments or by imaging them.

\section{B. Moment Fitting}

As discussed earlier, the 3D correlation function can be represented as a sum of independent cartesian moments. Thus, a straight forward procedure for source function extraction is to assume a shape and perform a simultaneous fit to all of the measured independent moments. The open squares in the top panel of Fig. 10 show the results of such a fit with an assumption of a 3D Gaussian source function (termed ellipsoid since there is no requirement that all three radii are the same). The fit parameters are indicated in panel (c). Overall, the moments are reasonably well represented by an ellipsoid for $\sqrt{s}=8.9$ $\mathrm{GeV}$. However, a clear deviation is apparent for $R_{y 2}^{2}$. A similar fit performed for the moments obtained for $\sqrt{s}=17.3 \mathrm{GeV}$ show even larger non Gaussian deviations.

The bottom panels of Fig. 10 show the results of a fit which assumes a linear combination of two Gaussians for the source function. The fit parameters are indicated in the figure. The two-source shape yields a much better representation of the data for $\sqrt{s}=17.3 \mathrm{GeV}$. The parameters obtained from these and the earlier fits, can be used to generate source functions in the out-, side- and long-directions for comparison with models.
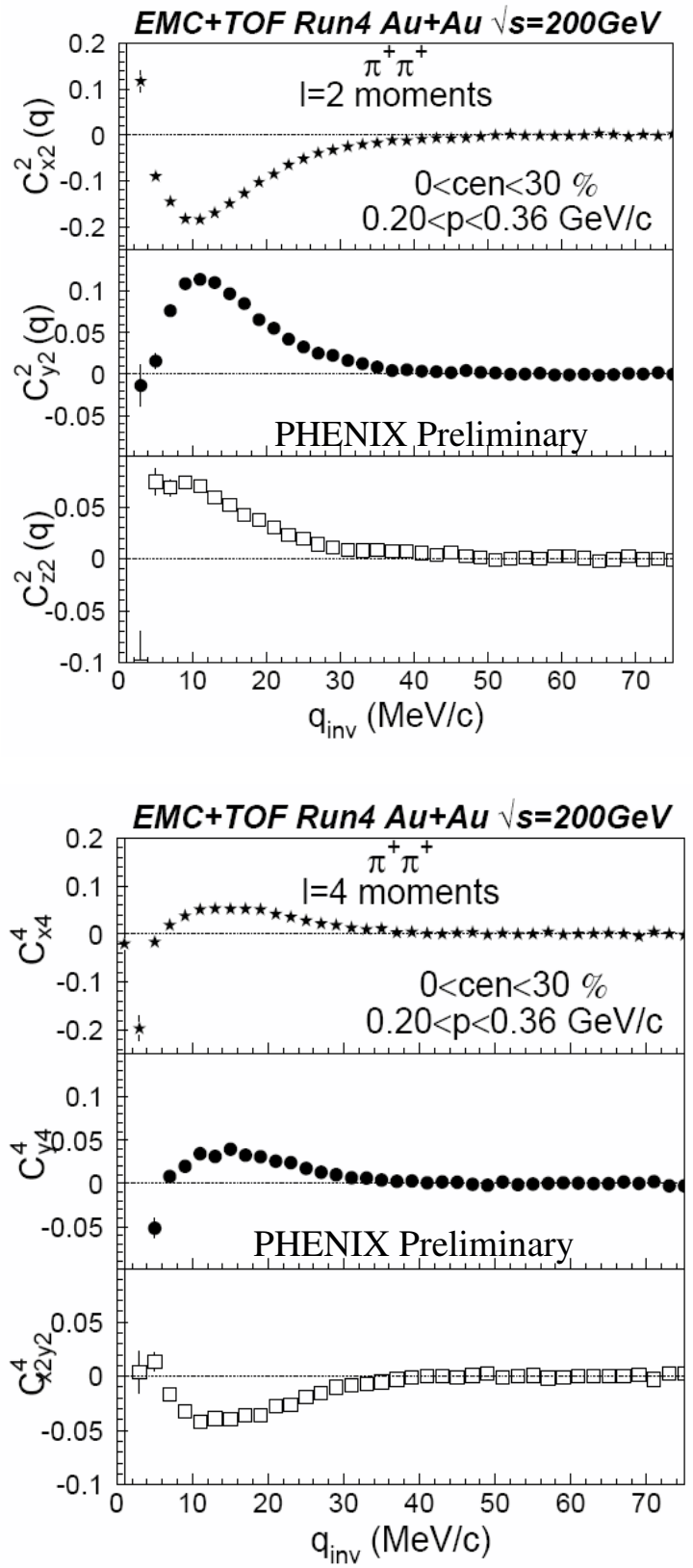

FIG. 9: $l=2$ (top panels) and $l=4$ (bottom panels) correlation moments for mid-rapidity (low $p_{T}$ ) $\pi^{-} \pi^{-}$pairs from $0-30 \%$ central $\mathrm{Au}+\mathrm{Au}$ collisions at $\sqrt{s}=200 \mathrm{GeV}$. Note the change in notation; $C_{\alpha_{1} \ldots \alpha_{l}}^{l}(q) \equiv R_{\alpha_{1} \ldots \alpha_{l}}^{l}(q)$.

\section{Moment Imaging}

A complimentary approach for obtaining the source function distributions is that of imaging. In this case, both the $3 \mathrm{D}$ correlation function $\mathrm{C}(\mathbf{q})$ and source function $\mathrm{S}(\mathbf{r})$ are expanded in a series of cartesian harmonic basis with correlation moments $R_{\alpha_{1} \ldots \alpha_{l}}^{l}(q)$ and source moments $S_{\alpha_{1} \ldots \alpha_{l}}^{l}(q)$ respec- 

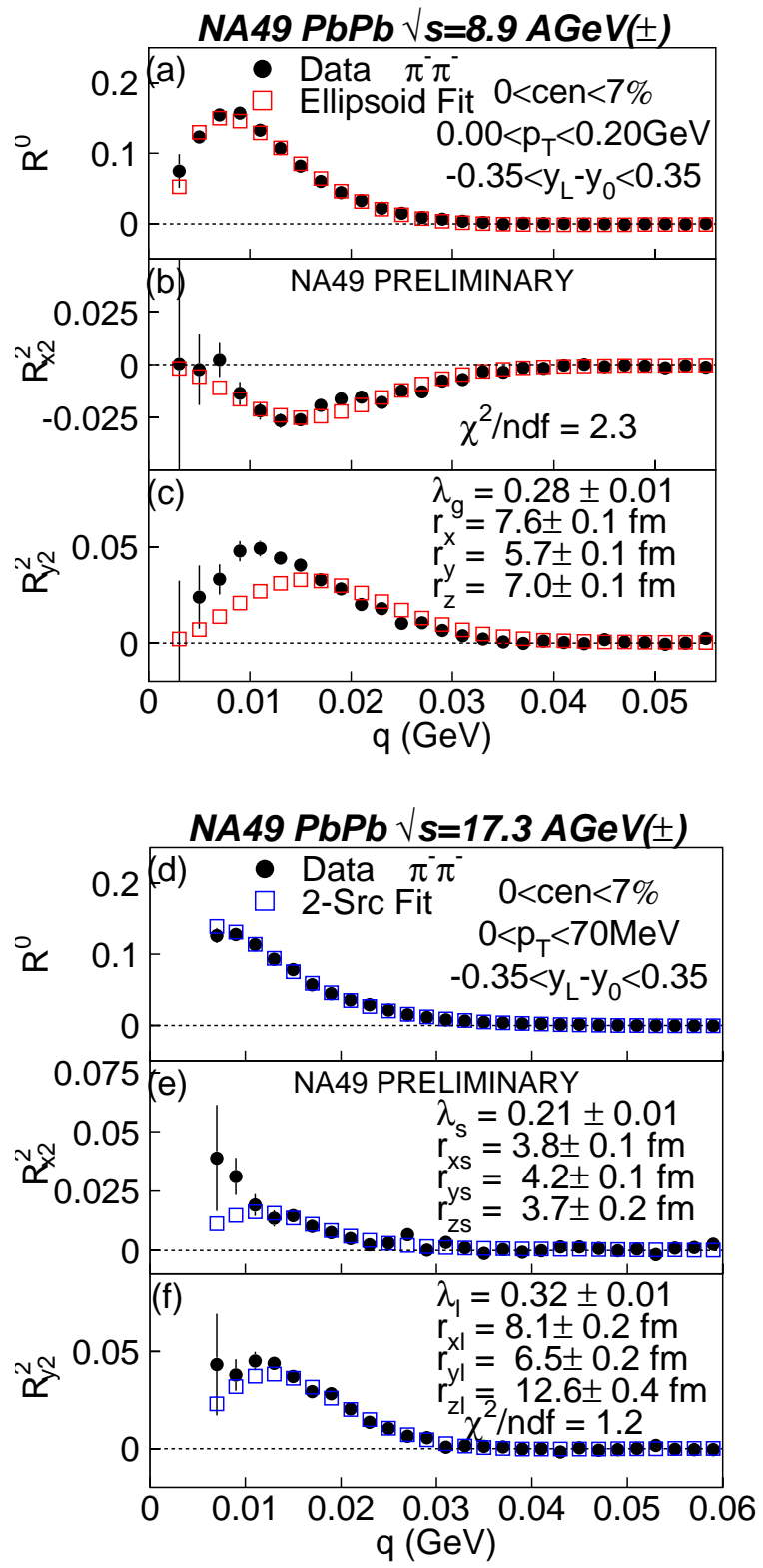

FIG. 10: (Color online) $l=2$ correlation moments for mid-rapidity, low $p_{T} \pi^{-} \pi^{-}$pairs from central $\mathrm{Pb}+\mathrm{Pb}$ collisions for $\sqrt{s}=8.9 \mathrm{GeV}$ (top panels) and $\sqrt{s}=17.3 \mathrm{GeV}$. The open squares represent the result of a simultaneous fit of the moments with an an assumed shape for the 3D source function (see text).

tively. Substitution into the 3D Koonin-Pratt equation

$$
C(\mathbf{q})-1=\int d \mathbf{r} K(\mathbf{q}, \mathbf{r}) S(\mathbf{r}),
$$

gives [52];

$$
R_{\alpha_{1} \ldots \alpha_{l}}^{l}(q)=4 \pi \int d r r^{2} K_{l}(q, r) S_{\alpha_{1} \ldots \alpha_{l}}^{l}(q),
$$

which relates the correlation moments $R_{\alpha_{1} \ldots \alpha_{l}}^{l}(q)$ to source moments $S_{\alpha_{1} \ldots \alpha_{l}}^{l}(q)$. Eq. 6 is similar to the 1D Koonin-Pratt equation (cf. Eq. 1) but pertains to moments describing different ranks of angular anisotropy $l$.

The mathematical structure of Eq.6 is similar to that of Eq. 1. Therefore, the same 1D Imaging technique (describe earlier) can be used to invert each correlation moment $R_{\alpha_{1} \ldots \alpha_{l}}^{l}(q)$ to extract the corresponding source moment $S_{\alpha_{1} \ldots \alpha_{l}}^{l}(q)$. Subsequently, the total 3D source function is calculated by combining the source moments for each $l$ as in equation (4)

$$
S(\mathbf{r})=\sum_{l} \sum_{\alpha_{1} \ldots \alpha_{l}} S_{\alpha_{1} \ldots \alpha_{l}}^{l}(r) A_{\alpha_{1} \ldots \alpha_{l}}^{l}\left(\Omega_{\mathbf{r}}\right)
$$
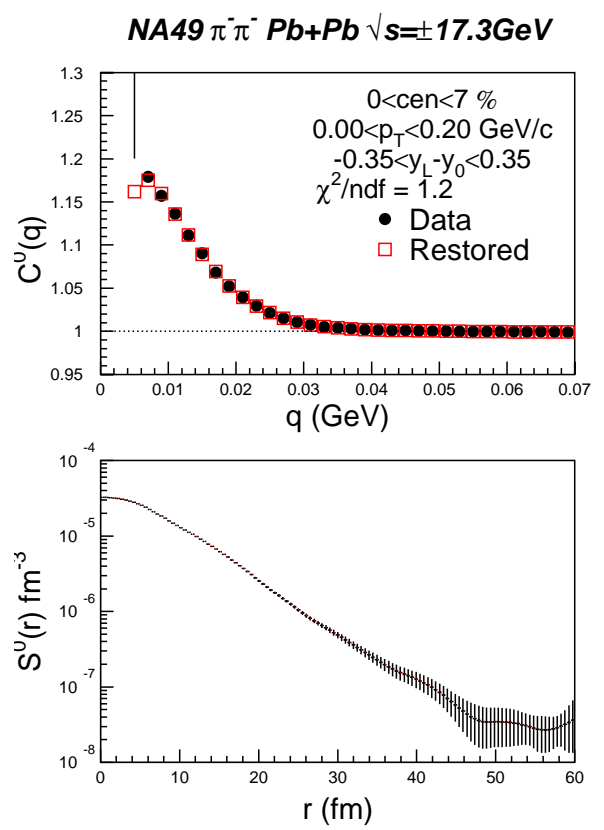

FIG. 11: (Color online) $1=0$ moment $C^{0}(q)=R^{0}(q)+1$ (top panel) and imaged source moment $S^{0}(r)$ (bottom panel) for mid-rapidity low $p_{T} \pi^{-} \pi^{-}$pairs from central $\mathrm{Pb}+\mathrm{Pb}$ collisions $(\sqrt{s}=17.3 \mathrm{GeV})$. The open squares in the top panel represent the correlation moment restored from the imaged source moment $S^{0}(r)$.

Figure 11 shows the imaged $l=0$ moment $S^{0}$ for the input $1=0$ moment $C^{0}(q)=R^{0}(q)+1$ for mid-rapidity low $p_{T}$ $\pi^{-} \pi^{-}$pairs from central $\mathrm{Pb}+\mathrm{Pb}$ collisions (bottom panel). This source image looks essentially exponential-like. Inserting the extracted image into the 1D Koonin-Pratt equation (Eq. 1) yields the restored moment shown as open squares in the top panel. The good agreement between the input and restored correlation moments serves as a consistency check of the imaging procedure.

The sources images for the $l=2$ moments $R_{x x}^{2}$ and $R_{y y}^{2}$ are shown in Figs. 12 and 13. They show that the anti-correlation exhibited by $R_{x x}^{2}$ results in a positive $l=2$ source moment contribution $S_{x x}^{2}$. That is, the negative contribution of $R_{x x}^{2}$ plus $R^{0}$ causes the overall correlation function to be narrower in the $x$ direction compared to the angle-average correlation function. A narrower correlation function in $x$ gives a broader source function in $x$; hence, the positive contribution of $S_{x x}^{2}$ to $S^{0}$. 
$N A 49 \pi^{-} \pi^{-} P b+P b \sqrt{ }= \pm 17.3 G e V$
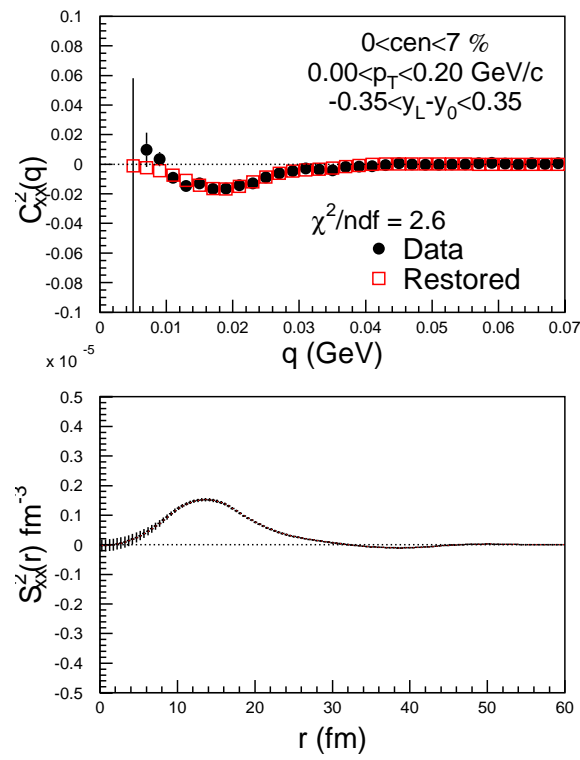

FIG. 12: (Color online) Same as Fig. 11 but for the $1=2$ moment $C_{x x}^{2}(q)=R_{x x}^{2}(q)$. Open squares in top panel represent correlation moment restored from imaged source moment $S_{x x}^{2}(r)$.

Figure 13 shows an opposite effect for the image for the $R_{y y}^{2}$ correlation moment. Here, the positive contribution of this correlation moment results in a negative contribution of the $l=2$ source moment $S_{y y}^{2}$. The positive contribution of $R_{y y}^{2}$ plus $R^{0}$ causes the overall correlation function to be broader in the $y$ direction compared to the angle-average correlation function. The resulting narrower source function (in $y$ ) reflects the negative contribution of $S_{y y}^{2}$ to $S^{0}$.

As discussed earlier, the imaged moments are combined to give the resulting pair separation distributions in the out-, sideand long-directions.

\section{Extracted source functions}

As discussed in the previous section,

$$
S(\mathbf{r})=\sum_{l} \sum_{\alpha_{1} \ldots \alpha_{l}} S_{\alpha_{1} \ldots \alpha_{l}}^{l}(r) A_{\alpha_{1} \ldots \alpha_{l}}^{l}\left(\Omega_{\mathbf{r}}\right) .
$$

Therefore, the net source functions in the $x$ (out-), $y$ (side-) and $z$ (long-) directions are simply given by the sum of the $1 \mathrm{D}$ source $S^{0}$ and the corresponding higher $l$ contributions $S_{i i}^{2}$ where $\mathrm{i}=\mathrm{x}, \mathrm{y}, \mathrm{z}$. Figs. $14-16$ show the source functions extracted for $\mathrm{Pb}+\mathrm{Pb}$ and $\mathrm{Au}+\mathrm{Au}$ collisions for the collision energies indicated. For the $\mathrm{Pb}+\mathrm{Pb}$ collisions, source moments were found to be significant only for the multi-polarities $l=0$ and $l=2$ for $0<p_{T}<70 \mathrm{MeV} / \mathrm{c}$. For $\mathrm{Au}+\mathrm{Au}\left(.2<p_{T}<.36\right.$ $\mathrm{GeV} / \mathrm{c})$ significant source moments were also obtained for $l=$ 4.

In Figs. 14 and 15 the results obtained from source imaging and fits to the correlation moments are shown. Clear indications for non-Gaussian contributions to these source functions
$N A 49 \pi^{-} \pi^{-} P b+P b \sqrt{ }= \pm 17.3 G e V$
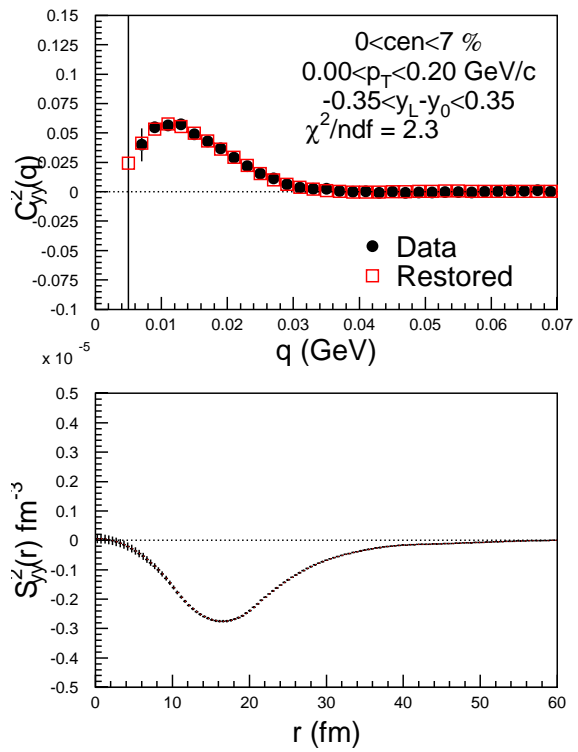

FIG. 13: (Color online) Same as Fig. 12 but for the $1=2$ moment $C_{y y}^{2}(q)=R_{y y}^{2}(q)$. Open squares in top panel represent correlation moment restored from imaged source moment $S_{y y}^{2}(r)$.

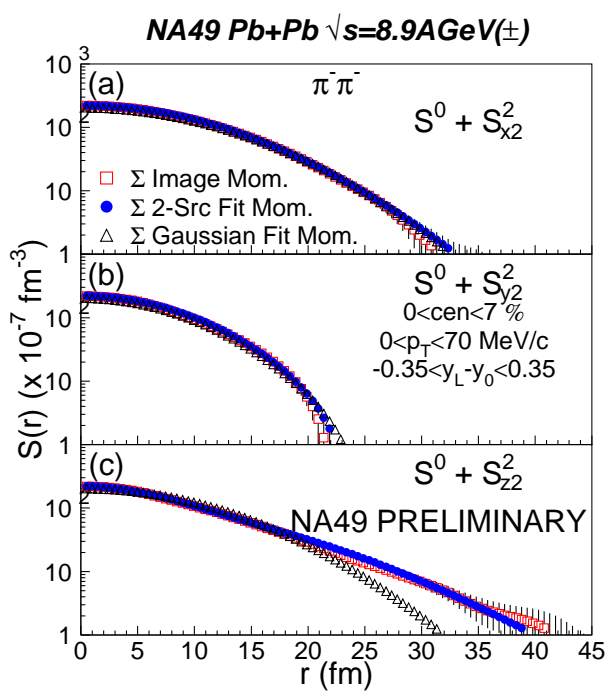

FIG. 14: (Color online) Total source function in (a) $x$ (out) (b) $y$ (side) (c) $z$ (long) direction for low $p_{T}$ mid-rapidity $\pi^{-} \pi^{-}$pairs from central $\mathrm{Pb}+\mathrm{Pb}$ collisions at $\sqrt{s}=8.9 \mathrm{GeV}$. Source image and fits are indicated.

are apparent. A detailed analysis of these source functions is still ongoing. However, a number of observations can be drawn. First, the ratio of the RMS radii of the source functions in the $x$ and $y$ directions are $1.3 \pm 0.1$ and and $1.2 \pm 0.1$ respectively. This deviation from unity points to a finite pion emission time. Here, it is important to emphasize that the rather low selection $0<p_{T}<70 \mathrm{MeV} / \mathrm{c}$, ensures that the Lorentz factor $\gamma$ is essentially 1 . That is, any extension in the $x$ direc- 


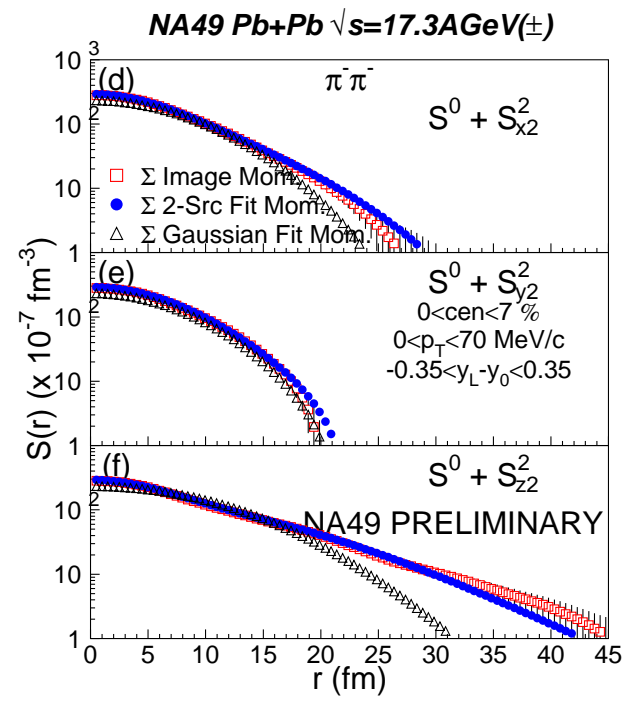

FIG. 15: (Color online) Same as Fig.14 but for $\sqrt{s}=17.3 \mathrm{GeV}$.

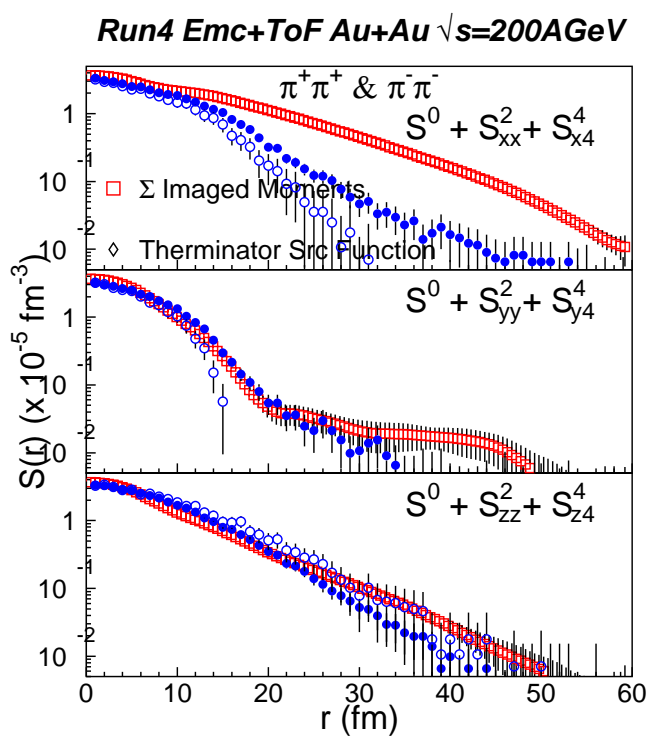

FIG. 16: (Color online) Source images for the $x$ (out) (a) $y$ (side) (b) and $z$ (long) (c) directions for $.2<p_{T}<.35 \mathrm{GeV} / \mathrm{c}$ mid-rapidity $\pi^{-} \pi^{-}$pairs from central Au+Au collisions at $\sqrt{s}=200 \mathrm{GeV}$. Model calculations from the Therminator code are also indicated. tion due to kinematic transformation from the LCMS to the PCMS is negligible.

Another important observation is that the RMS pair separation in the $z$ direction (cf. Figs. 14(c) and 15(f)) are $11 \mathrm{fm}$ and $12 \mathrm{fm}$ respectively. These dimensions are much larger than the Lorentz-contracted nuclear diameters of $3 \mathrm{fm}$ at $\sqrt{s}=8.9$ $\mathrm{GeV}$ and $1.5 \mathrm{fm}$ at $\sqrt{s}=17.3 \mathrm{GeV}$. In fact, they can be taken as the RMS pair separation resulting from the longitudinal spread of nuclear matter created by the passage of the two nuclei. Since the latter are moving with almost the speed of light, one can infer a lower bound "break-up" time for the created nuclear matter. This is estimated to be $\sim 8 \mathrm{fm} / \mathrm{c}$ at $\sqrt{s}=8.9$ $\mathrm{GeV}$ and $\sim 10 \mathrm{fm} / \mathrm{c}$ at $\sqrt{s}=17.3 \mathrm{GeV}$.

Figure 16 show representative source images extracted for $\mathrm{Au}+\mathrm{Au}$ collisions at $\sqrt{s}=200 \mathrm{GeV}$. For comparison, source functions obtained from initial calculations performed with the Therminator simulation code [54] are also plotted. The filled and open circles show the results from calculations with and without resonance emissions turned on. Fig. 16b shows that the calculations which include resonance emissions can account for an apparent tail in the distribution for the $y$ (side) direction. However, Fig. 16a suggests that such resonance contributions are not sufficient to account for an apparent extension in the $x$ (out) direction. An extensive effort to map out the source functions for different models and model parameters is currently underway. It is expected that a comparison between the results of such calculations and data will provide tighter constraints for the space-time evolution for collision systems spanning the AGS - SPS - RHIC bombarding energy range.

\section{CONCLUSIONS}

In summary, we have presented and discussed several techniques for femtoscopic measurements spanning collision energies from the AGS to RHIC. These methods of analysis provide a robust technique for detailed investigations of the reaction dynamics. Initial results from an ambitious program to measure a "full" femtoscopic excitation function, indicate that the emission source functions are decidedly non-Gaussian for a broad range of collision energies, and particle emission times can be reliably measured.
[1] F. Karsch et al., Phys. Lett. B 478, 447 (2000).

[2] QM2005, Nucl. Phys. A 774, 1c (2006).

[3] F. Karsch (2006), hep-lat/0601013.

[4] F. Karsch, E. Laermann, and A. Peikert, Nucl. Phys. B 605, 579 (2001), hep-lat/0012023.

[5] S. Pratt, Phys. Rev. Lett. 53, 1219 (1984).

[6] D. H. Rischke et al., Nucl. Phys. A 608, 479 (1996).

[7] M. A. Lisa et al., Annu. Rev. Nucl. Part. Sci. 55, 357 (2005).

[8] C. Adler et al., Phys. Rev. Lett. 87, 082301 (2001).
[9] K. Adcox et al., Phys. Rev. Lett. 88, 192302 (2002).

[10] J. Adams et al., Phys. Rev. Lett. 91, 262301 (2003).

[11] Y. Sinyukov et al., Phys. Lett. B 432, 248 (1998).

[12] R. Ganz et al. (The NA49) (1998), nucl-ex/9808006.

[13] I. G. Bearden et al. (NA44) (2003), nucl-ex/0305014.

[14] S. S. Adler et al., Phys. Rev. Lett. 93, 152302 (2004).

[15] T. Csorgo, S. Hegyi, T. Novak, and W. A. Zajc (2005), nuclth/0512060.

[16] K. Adcox et al., Nucl. Phys. A 757, 184 (2005), nucl- 
ex/0410003.

[17] J. Adams et al., Nucl. Phys. A 757, 102 (2005), nuclex/0501009.

[18] B. B. Back et al., Nucl. Phys. A 757, 28 (2005), nuclex/0410022.

[19] I. Arsene et al., Nucl. Phys. A 757, 1 (2005), nucl-ex/0410020.

[20] M. Gyulassy et al., Nucl. Phys. A 750, 30 (2005), nuclth/0405013.

[21] B. Müller (2004), nucl-th/0404015.

[22] E. V. Shuryak, Nucl. Phys. A 750, 64 (2005), hep-ph/0405066.

[23] U. Heinz et al., Nucl. Phys. A 702, 269 (2002).

[24] C. Adler et al. (STAR), Phys. Rev. Lett. 90, 082302 (2003), nucl-ex/0210033.

[25] S. S. Adler et al. (PHENIX), Phys. Rev. Lett. 97, 052321 (2006), nucl-ex/0507004.

[26] A. Adare (PHENIX) (2006), nucl-ex/0608033.

[27] M. Issah and A. Taranenko (PHENIX) (2006), nuclex/0604011.

[28] M. Csanád et al. (2005), nucl-th/0512078.

[29] M. Csanad et al. (2006), nucl-th/0605044.

[30] R. S. Bhalerao et al., Phys. Lett. B 627, 49 (2005).

[31] S. A. Voloshin, Nucl. Phys. A 715, 379 (2003).

[32] R. J. Fries et al., Phys. Rev. C 68, 044902 (2003).

[33] V. Greco et al., Phys. Rev. C 68, 034904 (2003).

[34] R. A. Lacey and A. Taranenko (2006), nucl-ex/0610029.

[35] N. Xu, Nucl. Phys. A 751, 109 (2005).

[36] B. Muller and J. L. Nagle (2006), nucl-th/0602029.

[37] S. S. Adler et al., Phys. Rev. Lett. 94, 232302 (2005).
[38] J.-W. Chen and E. Nakano (2006), hep-ph/0604138.

[39] P. Kovtun, D. T. Son, and A. O. Starinets, Phys. Rev. Lett. 94, 111601 (2005), hep-th/0405231.

[40] L. P. Csernai, J. I. Kapusta, and L. D. McLerran, Phys. Rev. Lett. 97, 152303 (2006), nucl-th/0604032.

[41] A. Nakamura and S. Sakai, Phys. Rev. Lett. 94, 072305 (2005), hep-lat/0406009.

[42] C. Nonaka and M. Asakawa, Phys. Rev. C 71, 044904 (2005), nucl-th/0410078.

[43] B. Kampfer, M. Bluhm, R. Schulze, D. Seipt, and U. Heinz (2005), hep-ph/0509146.

[44] J. I. Kapusta, P. Lichard, and D. Seibert, Phys. Rev. D 44, 2774 (1991).

[45] C. Gerschel and J. Hufner, Nucl. Phys. A 544, 513c (1992).

[46] S. S. Adler et al. (PHENIX) (2006), nucl-ex/0605032.

[47] S. E. Koonin, Phys. Lett. B 70, 43 (1977).

[48] D. A. Brown et al., Phys. Rev. C 64, 014902 (2001).

[49] D. A. Brown et al., Phys. Lett. B 398, 252 (1997).

[50] P. Chung et al., These proceedings (2006).

[51] S. Nickerson et al., Phys. Rev. C 57, $3251(1998)$.

[52] P. Danielewicz and S. Pratt, Phys. Lett. B 618, 60 (2005), nuclth/0501003.

[53] P. Chung, W. Holzmann, R. Lacey, J. Alexander, and P. Danielewicz, AIP Conf. Proc. 828, 499 (2006).

[54] A. Kisiel, W. Florkowski, and W. Broniowski (2006), nuclth/0602039. 